\title{
Suturing in Confined Spaces: Constrained Motion Control of a Hybrid 8-DoF Robot
}

\author{
Ankur Kapoor, Nabil Simaan, Russell H. Taylor \\ Department of Computer Science \\ Johns Hopkins University \\ Baltimore, MD \\ Email: \{kapoor,nsimaan,rht\}@cs.jhu.edu
}

\begin{abstract}
We present our work on developing and testing the high-level control for a future tele-robotic system for minimally invasive surgery of the throat and upper airways. As a testbed for these experiments, we used a hybrid 8 Degrees-ofFreedom (DoF) experimental robot composed of a six DoF robot and a two DoF snake-like unit. The kinematics and weighted redundancy resolution to support suturing in confined spaces, such as the throat, is developed and experimental validation in presented. The kinematics of the hybrid system is described in an 8-dimensional augmented vector space composed from the joint variables of the six DoF robot and two angles describing the configuration of the snake-like unit. Then a weighted, multi objective, optimization framework is used to perform the suturing operation under the assumption of a predefined suture geometry while satisfying joint limits, torque constraints, and minimizing extraneous motions of the system joints.
\end{abstract}

\section{INTRODUCTION}

Snake-like robots have been used extensively for various applications such as pipe inspection [1]-[3], search and rescue [4], [5], etc. In the last decade, these robots have been tested also for medical applications. While the majority of works focused on developing endoscopes and active bending catheters [6]-[11] there is a growing number of works targeting surgical assistance, including distal dexterity enhancement for Minimally Invasive Surgery (MIS). Our group is currently focusing on developing a tele-robotic system for MIS of the larynx and upper airways [12], [13].

The SLU is composed from an array of spacer disks affixed along a central super-elastic tube called the primary backbone. Three other super-elastic NiTi tubes are equally displaced around the central backbone and are called secondary backbones. These backbones are rigidly attached only to the end disk of the SLU. By using both push and pull forces on these backbones, the SLU can bend in any direction and the load distribution on the backbones can be optimized. This is achieved through a separate actuation redundancy algorithm as presented in [12]. The similarities and differences between this unit and previous works using flexible backbones and wire actuation were described in [12], [14].

Previous works on redundancy resolution used pseudoinverse control to provide dexterity optimization [15], [16], torque minimization [17], and singularity avoidance [16]. Other works used variational calculus for path planning [18] and control of hyper redundant robots [19], and singularity avoidance for parallel robots [20]. While these methods

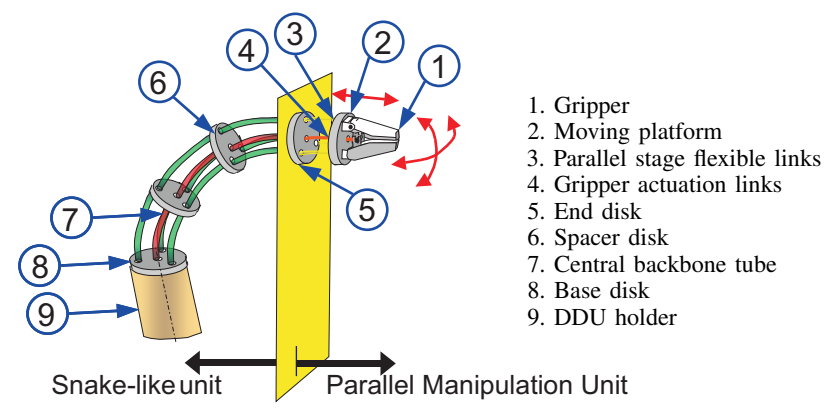

Fig. 1. The Distal Dexterity Unit (DDU) composed from a multi-backbone snake-like unit (SLU) equipped with a parallel tip

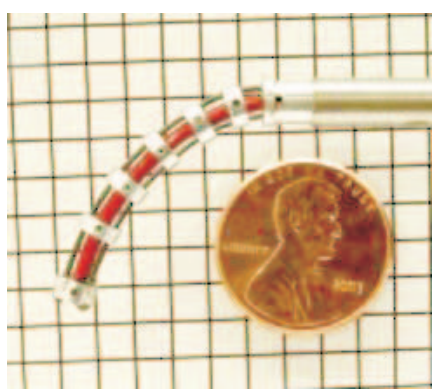

(a)

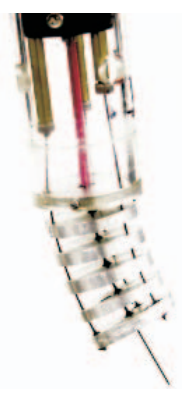

(b)
Fig. 2. Pictures of (a) $4 \mathrm{~mm}$ and (b) $27.6 \mathrm{~mm}$ snake-like units (SLU's) in a bent configuration

present two extremes of local and global optimization approaches, we focus our work on an optimization framework that is fast enough for online control and is easily modified to include obstacle avoidance, and virtual fixtures. We rely heavily on the work of Funda et al. [21] and Li and Taylor [22] in developing our control.

Fig. 2(a) presents the $4 \mathrm{~mm}$ SLU used to develop the DDU for the tele-robotic system for MIS of the throat. For this work, we attached a large-scale model (Fig. 2(b)) of the SLU to a modified version of the LARS - a 6-DoF robot developed at IBM [23], [24]. This robot is composed from a 3 DoF X-Y-Z stage that is serially attached to a "Remote Center of Motion" (RCM) mechanism. This mechanism is designed to rotate the tool tip around a fixed point in space. This is a highly desirable feature for MIS where the tool is limited to a fixed fulcrum point in the patient body. The axis of rotation of the third 
rotary joint passes through this remote point. Fig. 3 presents the LARS robot and the kinematic nomenclature used for its position analysis.

The following section outlines the kinematics of the SLU and the hybrid system composed from the LARS and the SLU of Fig. 4. Section III presents our approach for high-level control to support suturing in confined spaces. Section IV describes our validation setup and presents results of our experiments. Finally, section V summarizes the results of our experiments followed by conclusions.

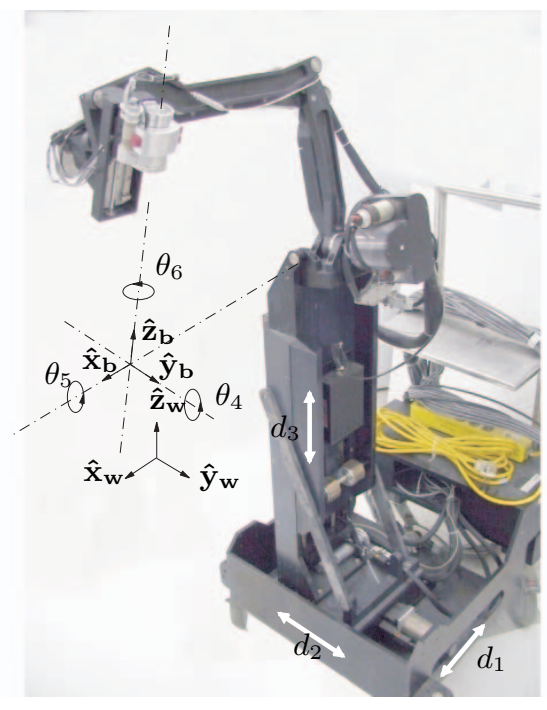

Fig. 3. The LARS robot and its kinematics

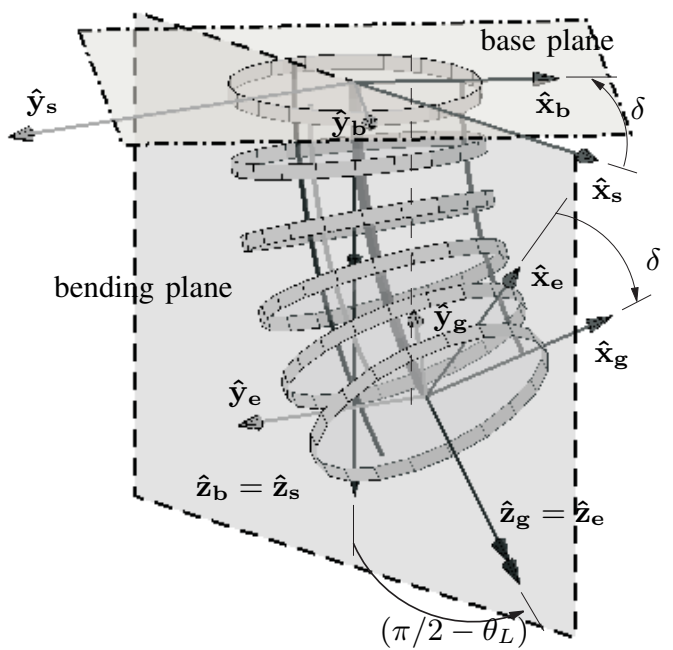

Fig. 4. The snake-like unit (SLU) and kinematic nomenclature

\section{Kinematic Model}

Fig. 3 and 4 show the nomenclature of LARS and SLU respectively. In this paper we assume that the SLU is attached to the LARS such that the origin of the base disk lies along this axis. We define the following coordinate frames to facilitate further discussion of the kinematics of the system: The gripper frame $\{g\}$, end disk frame $\{e\}$, snake plane frame $\{s\}$, base disk frame $\{b\}$ and the world frame $\{w\}$. Without loss of generality, we can assign the frames such that the world frame coincides with the base disk frame when all the joints are at a predefined home position.

The following notation is used in this paper:

$\{a\} \quad-$ A right handed coordinate frame named $a$.

$\mathbf{R} \mid \mathbf{p}] \quad$ - A homogeneous $4 \times 4$ transformation with rotation matrix $\mathbf{R}$ and a translational component $\mathbf{p}$.

${ }^{b} \mathbf{T}_{a} \quad$ - A homogeneous $4 \times 4$ transformation from frame $\{a\}$ to frame $\{b\}$.

$\mathbf{R}(\mathbf{a}, \alpha)$ - A rotation matrix about axis a by an angle $\alpha$.

$(\mathbf{a})^{\wedge} \quad-$ A Cross product matrix, such that $(a)^{\wedge} b=$ $a \times b$.

$\mathbf{p}_{a b}^{c} \quad-\quad$ A vector from $a$ to $b$ with respect to frame $\{c\}$.

$\mathbf{v}_{a b}^{c} \quad$ - Relative linear velocity of frame $\{b\}$ with respect to frame $\{a\}$ described in frame $\{c\}$.

$\boldsymbol{\omega}_{a b}^{c} \quad$ - Relative angular velocity of frame $\{b\}$ with respect to frame $\{a\}$ described in frame $\{c\}$.

$\hat{\mathbf{x}}_{\mathbf{a}}, \hat{\mathbf{y}}_{\mathbf{a}}, \hat{\mathbf{z}}_{\mathbf{a}}$ - Unit vectors associated with coordinate frame $\{a\}$.

$d_{1}, d_{2}, d_{3}$ - The joint variable for translational joint of LARS.

$\theta_{4}, \theta_{5}, \theta_{6}$ - The joint variable for rotational joint of LARS.

$L \quad$ - Length of SLU primary backbone.

$r \quad$ - Radius of base, space and end disks.

$\theta(s) \quad-$ The angle of primary backbone tangent in $\hat{\mathbf{x}}_{\mathbf{1}}$ $\hat{\mathbf{z}}_{1}$ plane. $\theta_{0}=\theta(s=0)$ and $\theta_{L}=\theta(s=L)$.

$\delta \quad-\quad$ The rotation angle of snake plane about $\hat{\mathbf{z}}_{\mathrm{b}}$.

$\beta \quad-$ Division angle. $\beta=2 \pi / k . k$ is the number of secondary backbone.

$i \quad-$ Index of the secondary backbone of SLU, $i=1,2,3$.

$L_{i} \quad$ - Length of $i^{\text {th }}$ backbone.

The direct kinematics for the LARS robot is as follows

$$
\begin{aligned}
{ }^{u} \mathbf{T}_{b}= & {\left[\mathbf{I} \mid d_{1} \hat{\mathbf{x}}_{\mathbf{w}}\right]\left[\mathbf{I} \mid d_{2} \hat{\mathbf{y}}_{\mathbf{w}}\right]\left[\mathbf{I} \mid d_{3} \hat{\mathbf{z}}_{\mathbf{w}}\right] } \\
& \times\left[\mathbf{R}\left(\hat{\mathbf{y}}_{\mathbf{w}}, \theta_{4}\right) \mid \mathbf{0}\right]\left[\mathbf{R}\left(\hat{\mathbf{x}}_{\mathbf{w}}, \theta_{5}\right) \mid \mathbf{0}\right] \\
& \times\left[\mathbf{I} \mid \mathbf{p}_{\text {offset }}\right]\left[\mathbf{R}\left(\hat{\mathbf{x}}_{\mathbf{w}}, \pi\right) \mid \mathbf{0}\right]\left[\mathbf{R}\left(\hat{\mathbf{z}}_{\mathbf{w}}, \theta_{6}\right) \mid \mathbf{0}\right]
\end{aligned}
$$

The generalized twist ${ }^{1}$ of the frame $\{b\}, \dot{\mathbf{x}}_{b}^{w}$ is related to the joint velocities $\dot{\mathbf{q}}_{\text {lars }} \in \mathbb{R}^{6}$ of the LARS robot according to $(2)$

$$
\dot{\mathbf{x}}_{b}^{w}=\mathbf{J}_{\text {lars }} \dot{\mathbf{q}}_{\text {lars }}=\left[\begin{array}{l}
\mathbf{J}_{\text {vlars }} \\
\mathbf{J}_{\omega \text { lars }}
\end{array}\right] \dot{\mathbf{q}}_{\text {lars }}
$$

where $\mathbf{J}_{\text {lars }} \in \mathbb{R}^{6 \times 6}$ is the instantaneous direct kinematics Jacobian of the LARS robot.

\footnotetext{
${ }^{1}$ Twist is redefined in this paper such that the linear velocity component precedes the angular velocity component.
} 
The direct kinematics and the instantaneous kinematics of the SLU were presented in [12]. The same notation is used in this paper and part of it is presented here for completeness. As in [12], the pose of the SLU is represented by two angles $\theta_{L}$ and $\delta$. The joint variables for the SLU, that is the secondary backbone lengths depend on the angles $\theta_{L}$ and $\delta$ and are given by the inverse kinematic solution

$$
L_{i}=L+r\left(\theta_{L}-\theta_{0}\right) \cos (\delta)
$$

The position, $\mathbf{p}_{g b}^{b}$, and the orientation, ${ }^{b} \mathbf{R}_{g}$, of the end disk are obtained by integrating along the tangent of the backbone and by successive rotation sequence as in (4).

$$
\begin{aligned}
\mathbf{p}_{g b}^{b} & =\mathbf{R}\left(\hat{\mathbf{z}}_{\mathbf{b}},-\delta\right) \mathbf{p}_{g b}^{s} \\
{ }^{b} \mathbf{R}_{g} & =\mathbf{R}\left(\hat{\mathbf{z}}_{\mathbf{b}},-\delta\right) \mathbf{R}\left(\hat{\mathbf{y}}_{\mathbf{s}}, \pi / \mathscr{D}-\theta_{L}\right) \mathbf{R}\left(\hat{\mathbf{z}}_{\mathbf{e}}, \delta\right)
\end{aligned}
$$

where

$$
\mathbf{p}_{g b}^{s}=\left[\int_{0}^{L} \cos (\theta(s)) d s, \quad 0, \quad \int_{0}^{L} \sin (\theta(s)) d s\right]^{t}
$$

For small SLU where gravity effects are negligible, $\theta(s)$ will be a circular section of length $L$ and radius $\rho$ [25]. Thus $\theta(s)$ can be approximated by a linear function given by

$$
\theta(s)=\pi / 2+a s ; \text { where, } a=\left(\theta_{L}-\pi / 2\right) / L
$$

and (5) becomes

$$
\mathbf{p}_{g b}^{s}=\left\{\begin{array}{l}
{\left[\rho\left(\sin \left(\theta_{L}\right)-1\right), \quad 0, \quad \rho \cos \left(\theta_{L}\right)\right]^{t}, \theta \neq \pi / 2} \\
{\left[\begin{array}{ll}
0, & 0, \quad L
\end{array}\right]^{t}, \theta=\pi / 2}
\end{array}\right.
$$

By defining $\dot{\psi} \triangleq\left(\dot{\theta}_{L}, \quad \dot{\delta}\right)^{t}$, and differentiating (4) with respect to time, one obtains the linear and angular velocity of frame $\{g\}$ with respect to frame $\{b\}$ as

$$
\begin{aligned}
\mathbf{v}_{g}^{b} & =\mathbf{R}\left(\hat{\mathbf{z}}_{\mathbf{b}}, \delta\right)\left[\begin{array}{lll}
\dot{\mathbf{p}}_{g b}^{s}, & \mathbf{p}_{g b}^{s} \dot{\delta}, & \dot{\mathbf{p}}_{g b}^{s}
\end{array}\right]^{t} \\
\boldsymbol{\omega}_{g}^{b} & =-\dot{\delta} \hat{\mathbf{z}}_{\mathbf{s}}-\dot{\theta}_{L} \hat{\mathbf{y}}_{\mathbf{s}}+\dot{\delta} \hat{\mathbf{z}}_{\mathbf{e}}
\end{aligned}
$$

Equations (8) can be rearranged to give kinematic Jacobian relating the generalized twist $\dot{\mathbf{x}}_{g}^{b}$ with the angular rates $\dot{\psi}$.

$$
\dot{\mathbf{x}}_{g}^{b}=\mathbf{J}_{s l u} \dot{\boldsymbol{\psi}}=\left[\begin{array}{l}
\mathbf{J}_{v s l u} \\
\mathbf{J}_{\omega s l u}
\end{array}\right] \dot{\boldsymbol{\psi}} ; \quad \mathbf{J}_{s l u} \in \mathbb{R}^{6 \times 2}
$$

A frame transformation can be applied to the position and orientation of gripper frame $\{g\}$ to get direct and instantaneous kinematics in the world frame $\{w\}$. The final results are presented in (10) and (11).

$$
\begin{gathered}
{ }^{u} \mathbf{T}_{g}={ }^{u} \mathbf{T}_{b}\left[{ }^{b} \mathbf{R}_{g} \mid \mathbf{p}_{g b}^{b}\right] \\
\mathbf{v}_{g}^{w}=\mathbf{v}_{b}^{w}+{ }^{w} \mathbf{R}_{b} \mathbf{v}_{g b}^{b}+\left(\boldsymbol{\omega}_{b}^{w}\right)^{\wedge}\left({ }^{w} \mathbf{R}_{b} \mathbf{p}_{g b}^{b}\right) \\
\boldsymbol{\omega}_{g}^{w}=\boldsymbol{\omega}_{b}^{w}+{ }^{w} \mathbf{R}_{b} \boldsymbol{\omega}_{g b}^{b}
\end{gathered}
$$

The kinematics of hybrid system consisting of the 6-DoF LARS and 2-DoF SLU can be described using 8 independent variables. For convenience we define an augmented state vector $\mathbf{s} \in \mathbb{R}^{8}$ as composed of the joint variable of LARS and two angles describing the configuration of the SLU, that is, $\mathbf{s} \triangleq\left[\begin{array}{ll}\mathbf{q}_{\text {lars }}{ }^{t}, & \psi^{t}\end{array}\right]^{t}$. Such a selection of variables allows us to use (2), (8), and $\mathbf{x}_{b}^{w}=\left[\begin{array}{ll}\mathbf{v}_{b}^{w t}, & \boldsymbol{\omega}_{b}^{w t}\end{array}\right]^{t}$ in (11) to give a linear equation (12) in $\dot{\mathrm{s}}$.

$$
\dot{\mathbf{x}}_{g}^{w}=\mathbf{J}_{x s} \dot{\mathbf{s}}=\left[\begin{array}{l}
\mathbf{J}_{v} \\
\mathbf{J}_{\omega}
\end{array}\right] \dot{\mathbf{s}} ; \quad \mathbf{J}_{x s} \in \mathbb{R}^{6 \times 8}
$$

The joint velocities of the SLU are related to the angular rates $\dot{\psi}$ according to (13).

$$
\dot{\mathbf{q}}_{s l u}=\mathbf{J}_{l \psi} \dot{\boldsymbol{\psi}} ; \quad \mathbf{J}_{l \psi} \in \mathbb{R}^{3 \times 2}
$$

$\mathbf{J}_{l \psi}$ is found by taking the time derivative of (3) for $L_{i}=$ $1,2,3$.

\section{PATH PLANNING AND REDUNDANCy RESOlution FOR SUTURING IN CONFINED SPACES}

\section{A. Suture Model}

Using the kinematic nomenclature of previous section, we can explore different approaches for suturing in confined spaces. Fig. 5 shows a typical suture used during surgery. It is a circular section of known length and radius. For this paper, we assume that the suture is attached to the SLU such that the plane of the suture is parallel to the end disk and the center of the suture lies along the $\hat{\mathbf{z}}_{\mathrm{g}}$ axis of the end disk of the SLU. We also assume that the suture geometry is planar and known in advance; particularly we assume it to be circular. To minimize tissue tearing we propose to control the movement of the LARS and SLU such that the relative velocity of the needle with respect to the tissue is tangent to the needle at the point at which it pierces the tissue. For a circular suture this simplifies to performing a rotational motion about the center of the suture.

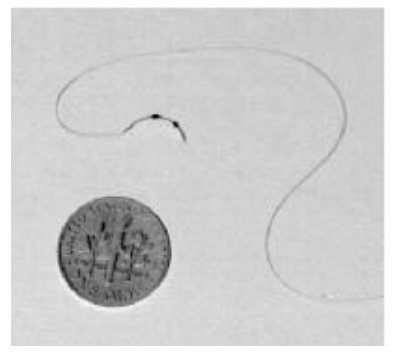

Fig. 5. A typical $13 \mathrm{~mm}, 3 / 8$ circle suture used in surgeries

\section{B. Redundancy Resolution for Suturing}

In this section, we discuss the implementation of our method for the task of suturing within a confined space. We assume that the target is specified and the robot is positioned such that the axis and center of the suture are aligned with the desired point and axis. We will use the augmented state vector $\mathbf{s}_{\text {start }}$ to represent the state of the robot at the beginning of the suture-biting-motion. The pose of frame $\{g\}$ with respect to world frame $\{w\}$ at this instance is represented by known 
position and orientation $\mathbf{p}_{g}^{w}$ start and ${ }^{w} \mathbf{R}_{g_{\text {start }}}$. We would like to perform path generation for suturing while minimizing tissue tear, avoiding maximal joint speeds, and avoiding joint limits. Moreover it is desirable to perform this task with minimum motion of the LARS and SLU joints, as this would allow multiple such units to operate while reducing the computational effort for collision avoidance amongst several tools inside the same confined space such as the throat. We chose to linearize our system by assuming the current position and planning for the next target position after a small time increment $\Delta T$. Hence we approximate velocities as $\dot{\mathbf{s}}=\Delta \mathbf{s} / \Delta T$ and $\dot{\mathbf{x}}=\Delta \mathbf{x} / \Delta T$. The outline of the algorithm is as follows: 1) Obtain the incremental motion desired by the user through force sensor, joystick or master 2) Formulate a set of linear constraints based on current robot state and specified task 3) Use the robot and task instantaneous kinematics to generate a quadratic program with linear constraints. The general form of the program is

$$
\begin{aligned}
\arg \min _{\Delta \mathbf{s} / \Delta T} & \left\|W\left(\Delta \mathbf{x}-\Delta \mathbf{x}_{\text {desired }}\right) / \Delta T\right\|, \\
\text { s.t. } & H \Delta \mathbf{x} / \Delta T \geq \mathbf{h}, \\
& \Delta \mathbf{x} / \Delta T=\mathbf{J}_{x s} \Delta \mathbf{s} / \Delta T
\end{aligned}
$$

where $\Delta \mathbf{s}$ is the desired incremental motions of the augmented state variables, $\Delta \mathbf{x}_{\text {desired }}, \Delta \mathrm{x}$ are the desired and the computed incremental motions of the task variables in Cartesian space, respectively. $\mathbf{J}_{\mathbf{x} \mathbf{s}}$ is the Jacobian matrix relating Cartesian space to augmented state vector space, $W$ is a diagonal matrix for weights. Equation (14b) forms a convex polyhedron which represents the feasible region for Cartesian velocities. Equation (14c) represents the kinematic constraints of the robot. 4) Solve the quadratic program for the incremental motion, which is used to move the robot. We would like to note that the constraints of step 3 might not be linear such as the distance function. In such cases we use a linear approximation, which allows us to utilize the structure of least squares problem with linear constraints, and solve the quadratic program in time frames suitable for robot control. We have used the Lawson and Hanson's algorithm as presented in [26].

1) Minimizing tissue tear: To ensure pure rotation about the center of the suture, we rotate the gripper such that its angular velocity vector is perpendicular to the suture plane and the center point of the suture is constrained to lie within a small sphere of radius $\epsilon_{g}$. This translates into the constrained optimization problem given by (15).

$$
\begin{aligned}
\arg \min _{\dot{\mathbf{x}}} & \left\|\mathbf{W}_{g}\left(\dot{\mathbf{x}}_{g}^{w}-\dot{\mathbf{x}}_{g \text { desired }}^{w}\right)\right\| \\
\text { s.t. } & \left\|\mathbf{p}_{g}^{w}-\mathbf{p}_{g \text { start }}^{w}\right\| \leq \epsilon_{g}
\end{aligned}
$$

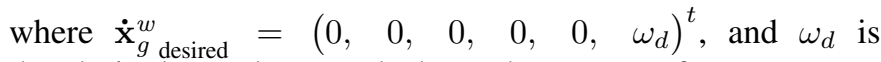
the desired angular speed about the center of suture. Here $W_{g} \in \mathbb{R}^{6 \times 6}$ is a diagonal matrix of weights. We would like to reformulate (15) so that it is compatible with (14) this can be achieved by replacing the non-linear Euclidean norm in (15b) by a linear approximation. We obtain such an approximation by bounding all possible projections of $\mathbf{p}_{g}^{w}$ start on all the radial unit vectors inside the unit sphere to be smaller than $\epsilon_{g}$. We then discritize the bundle of radial unit vectors as represented in the rows of the matrix $\mathbf{M}$, given by (16).

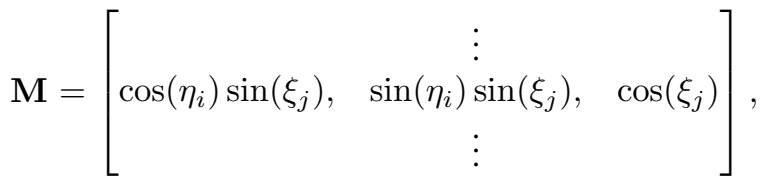

$$
\begin{aligned}
& i=1, \ldots, n ; j=1, \ldots, m
\end{aligned}
$$

where $\eta_{i}=i(2 \pi / n)$ and $\xi_{j}=j(2 \pi / n)$ represent the direction angles with respect to $\hat{\mathbf{x}}_{\mathbf{w}}$ and $\hat{\mathbf{z}}_{\mathbf{w}}$, respectively.

If we consider a sufficient number of unit vectors in $\mathbf{M}$ in different directions with $\mathbf{p}_{g}^{w}$ start as origin, (15b) can be approximated by a set of $n \times m$ linear inequalities given by (17).

$$
\mathbf{M}\left(\mathbf{p}_{g}^{w}-\mathbf{p}_{g \text { start }}^{w}\right) \leq \epsilon_{g} \mathbf{e}
$$

where $\mathbf{e}=(1, \cdots, 1)^{t} \in \mathbb{R}^{n m}$. Since $\mathbf{p}_{g}^{w}$ and $\mathbf{p}_{g}^{w}$ start are separated by a small time increment $\Delta T$, we approximate the velocity $\dot{\mathbf{p}}_{g}^{w}$ by $\left(\mathbf{p}_{g}^{w}-\mathbf{p}_{g}^{w}{ }_{\text {start }}\right) / \Delta T$. By using this approximation and (17), (15) can be written in the framework of (14) as:

$$
\begin{aligned}
\arg \min _{\dot{\mathbf{s}}} & \left\|\mathbf{W}_{g}\left(\dot{\mathbf{x}}_{g}^{w}-\dot{\mathbf{x}}_{g \text { desired }}^{w}\right)\right\| \\
\text { s.t. } & \mathbf{H}_{g} \dot{\mathbf{p}}_{g}^{w} \geq \mathbf{h}_{g} \\
& \dot{\mathbf{x}}_{g}^{w}=\mathbf{J}_{x s} \dot{\mathbf{s}}
\end{aligned}
$$

where $\mathbf{H}_{g}=-\mathbf{M}$ and $\mathbf{h}_{g}=-\left(\epsilon_{g} / \Delta T\right) \mathbf{e}$. Physically (18b) represents a polyhedron with $\mathrm{nm}$ vertices which we have used to approximate (15b).

2) Avoiding joint speed limits: To keep the actuation unit of the SLU compact, it was designed with small motors having limited power. This places limits on the joint velocities that can be attained by the SLU which can be stated as

$$
\dot{\mathbf{q}}_{\text {slu lo }} \leq \dot{\mathbf{q}}_{\text {slu }} \leq \dot{\mathbf{q}}_{\text {slu } u p}
$$

where $\dot{\mathbf{q}}_{\text {slu lo }}$ and $\dot{\mathbf{q}}_{\text {slu up }}$ are lower and upper limits for joint velocities attainable by the SLU secondary backbones respectively. We can use the following matrix representation for (19).

$$
\begin{aligned}
\arg \min _{\dot{\mathbf{s}}} & \left\|\mathbf{W}_{g}\left(\dot{\mathbf{x}}_{g}^{w}-\dot{\mathbf{x}}_{g \text { desired }}^{w}\right)\right\| \\
\text { s.t. } & \mathbf{H}_{t} \dot{\mathbf{q}}_{s l u} \geq \mathbf{h}_{t} \\
& \dot{\mathbf{x}}_{g}^{w}=\mathbf{J}_{x s} \dot{\mathbf{s}}
\end{aligned}
$$

where $\mathbf{H}_{t}=(\mathbf{I}, \quad-\mathbf{I})^{t} \in \mathbb{R}^{6 \times 3}$

$$
\text { and } \quad \mathbf{h}_{t}=\left(\dot{\mathbf{q}}_{\text {slu lo }}, \quad-\dot{\mathbf{q}}_{\text {slu up }}\right)^{t} \in \mathbb{R}^{6}
$$


3) Avoiding joint limits: To ensure that the motion is within the workspace of the system given by $\mathbf{s}_{l o}$ and $\mathbf{s}_{u p}$, we have to satisfy the following condition

$$
\mathbf{s}_{L} \triangleq \mathbf{s}_{l o}-\mathbf{s} \leq \dot{\mathbf{s}} \Delta T \leq \mathbf{s}_{u p}-\mathbf{s} \triangleq \mathbf{s}_{U}
$$

In addition, we would also like to minimize extraneous motion of the system joints to allow working in confined spaces. We can add an objective function with weight $\mathbf{W}_{s} \in \mathbb{R}^{8 \times 8}$, minimizing the individual rate of state change, $\dot{\mathbf{s}}$. It is of the form

$$
\arg \min _{\dot{\mathbf{s}}}\left\|\mathbf{W}_{s} \dot{\mathbf{s}}\right\|
$$

Equation (22) can be as

$$
\begin{aligned}
\mathbf{H}_{s} \dot{\mathbf{s}} & \geq \mathbf{h}_{s} \\
\text { where } \quad \mathbf{H}_{s} & =(\mathbf{I}, \quad-\mathbf{I})^{t} \in \mathbb{R}^{16 \times 8} \\
\text { and } \quad \mathbf{h}_{s} & =\left(\mathbf{s}_{L} / \Delta T, \quad \mathbf{s}_{U} / \Delta T\right)^{t} \in \mathbb{R}^{16}
\end{aligned}
$$

We shall represent the elements in vectors $\mathbf{s}_{L}$ and $\mathbf{s}_{U}$ that correspond to the SLU by $\mathbf{s}_{L s l u}$ and $\mathbf{s}_{U \text { slu }}$ respectively and these can be defined by using the desired maximal SLU speeds $\dot{\boldsymbol{\psi}}_{\max }$ such that $\mathrm{s}_{L \text { slu }}=-\dot{\boldsymbol{\psi}}_{\max } \Delta T$ and $\mathrm{s}_{U \text { slu }}=\dot{\boldsymbol{\psi}}_{\max } \Delta T$.

To summarize, cases 1-3 described above are all special cases of (14). Explicitly, (14) can be reformulated by combining all the minimized objective functions and constraints, substituting the equality constraint of (14c) and using (12) and (13) to give a combined optimization problem as given by (25).

$$
\begin{array}{r}
\arg \min _{\dot{\mathbf{s}}}\left\|\left[\begin{array}{cc}
\mathbf{W}_{g} & \mathbf{0} \\
\mathbf{0} & \mathbf{W}_{s}
\end{array}\right]\left(\left[\begin{array}{c}
\mathbf{J}_{x s} \\
\mathbf{I}
\end{array}\right] \dot{\mathbf{s}}-\left[\begin{array}{c}
\dot{\mathbf{x}}_{g}^{w} \\
\mathbf{0}_{\text {desired }}
\end{array}\right]\right)\right\| \\
\text { s.t. }\left[\begin{array}{ccc}
\mathbf{H}_{g} & \mathbf{0} & \mathbf{0} \\
\mathbf{0} & \mathbf{H}_{t} & \mathbf{0} \\
\mathbf{0} & \mathbf{0} & \mathbf{H}_{s}
\end{array}\right]\left[\begin{array}{cc}
\mathbf{J}_{v} \\
\mathbf{0} & \mathbf{J}_{l \psi}
\end{array}\right] \dot{\mathbf{s}} \geq\left[\begin{array}{l}
\mathbf{h}_{g} \\
\mathbf{h}_{t} \\
\mathbf{h}_{s}
\end{array}\right]
\end{array}
$$

The strength of this approach is that a large number of constraints and objective function can be easily incorporated. We can write all the constraints in terms of the state vector $\dot{\mathbf{s}}$ by using the appropriate Jacobian matrix. This problem can easily be solved numerically for the vector of state velocities s using methods presented in [26].

In (25a), the diagonal weight matrix $\mathbf{W}_{g}$ determines the "penalty" for error in the desired trajectory while $\mathbf{W}_{s}$ determines the "penalty" for high joint speeds of the LARS and high bending rates of the SLU. These weight matrices have different units; therefore, they are difficult to determine. Yoshikawa [27] proposed using normalized velocities given by $\hat{\mathbf{q}}_{i}=\mathbf{q}_{i} / q_{i \max }$ and $\hat{\mathbf{v}}_{j}=\mathbf{v}_{j} / v_{j \max }$ to overcome the problem of different units. In our work, we chose to use the method of Funda et al. [21] that scales weights such that the penalties for a $1 \mathrm{~mm}$ positional error and a $1 \mathrm{deg}$ rotational error are equal. Accordingly, we used $w_{\text {translational }}=(\pi / 180) \times w_{\text {rotational }}$ and empirically determined the weights based on simulations. The results of these simulations are presented in the following section.

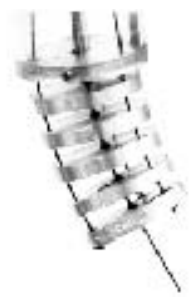

(a)

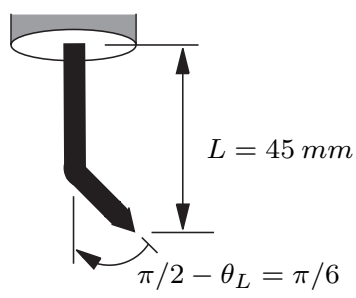

(b)
Fig. 6. Pictures of (a) SLU with a large and (b) equivalent bent rigid tool
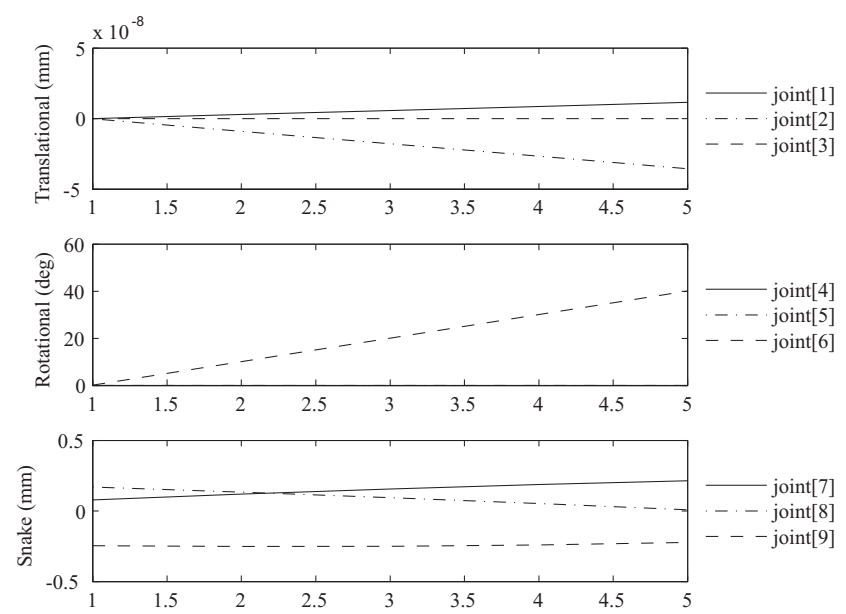

Fig. 7. Joint motions for test trajectory corresponding to $\mathbf{W}_{g}=$ $\operatorname{diag}\left\{1^{3}, 1^{3}, 1^{3}, 1^{3}, 1^{3}, 1^{3}\right\}$ and

$\mathbf{W}_{s}=\operatorname{diag}\{1,1,1,0.1 \pi / 180,0.1 \pi / 180,0.1 \pi / 180,0.01 \pi / 180$, $0.01 \pi / 180\}$

\section{Simulations And ExPERimental Results}

We performed two sets of simulations to determine the efficacy of suturing in confined spaces. We used the SLU for the first set of simulation and a bent rigid tool for the second set of simulations. The shape and size of the rigid tool were determined to be equivalent to the shape of the backbone of the SLU in a bent configuration, Fig 6.

A goal trajectory was given such that the suture is rotated about its center (as described in section III-A). The desired angular velocity of the suture was 1 degree/second about $\hat{\mathbf{z}}_{\mathrm{g}}$ with zero translational velocity of its center. The weight matrices $\mathbf{W}_{s}$ and $\mathbf{W}_{g}$ were empirically determined and set to $\mathbf{W}_{g}=\operatorname{diag}\left\{1^{3}, 1^{3}, 1^{3}, 1^{3}, 1^{3}, 1^{3}\right\}$ and $\mathbf{W}_{s}=\operatorname{diag}\{1,1,1,0.1 \pi / 180,0.1 \pi / 180,0.1 \pi / 180$, $0.01 \pi / 180,0.01 \pi / 180\}$.

Fig. 7 presents the results of the simulation for the case where the SLU is used to rotate the suture about its center. It is evident from this figure that the joints of the LARS do not move significantly, except for the last rotational stage that rotates the base of the snake. The radius of the sphere $\epsilon_{g}$ in (15) was set to $1^{-4} \mathrm{~mm}$. The center of the suture remained within $1^{-9} \mathrm{~mm}$ from its desired position.

Fig. 8 presents the same simulation for the case where the 

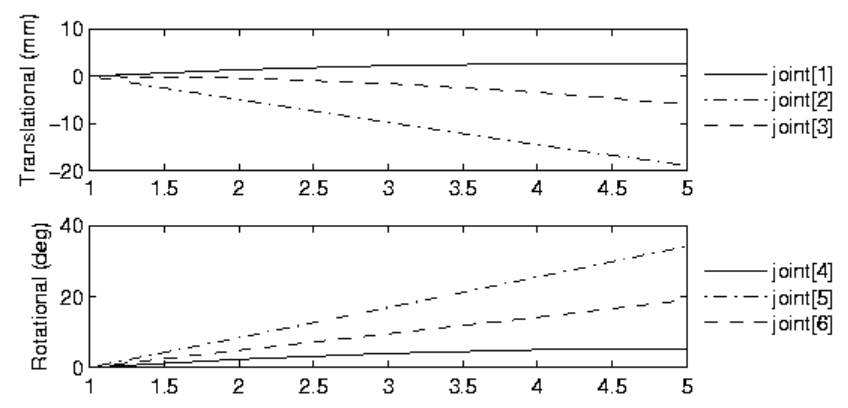

Fig. 8. Joint motions for test trajectory when using a rigid tool

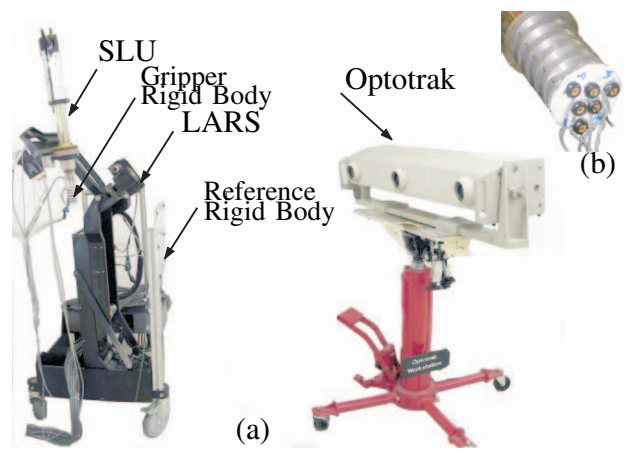

Fig. 9. Experimental setup showing (a) the LARS, the SLU Unit and the Optotrak and (b) a close-up of the SLU and gripper rigid body

suture is manipulated by the LARS while it is held by the rigid tool in Fig. 6(b). The results show that the joints of the LARS move significantly to compensate for the lack of distal dexterity. These motions are not possible for throat MIS since we have to manipulate several long tools passing through a single entry port (the patient's mouth). These results clearly indicate the importance of maintaining tool tip dexterity to avoid large motions in the proximal end of the tools.

\section{A. Experimental Setup}

Our experimental setup consists of the LARS robot and a large model of the SLU (Fig. 9). Though the final design of the robotic system for MIS is more compact and small, our current system was easy to fabricate and served as a robust platform for initial testing of our control. Three 4-Axis LoPoMoCo [28] cards provide I/O operations of reading encoders and providing analog outputs for motor voltage control. They also have on-board power amplifiers to control low power motors. An industrial PC (Pentium-II) houses these ISA cards and is used for the servo control and user interface. The PC runs a RealTime Application Interface for Linux (RTAI), thus providing crucial real-time functionality. Since the LoPoMoCo cards do not provide position servoing, a basic position servo (PID) is implemented in software. The optimized control algorithm is implemented under the assumption that motions within a single cycle are sufficiently small. Under this assumption, the instantaneous kinematic relationship between state velocities

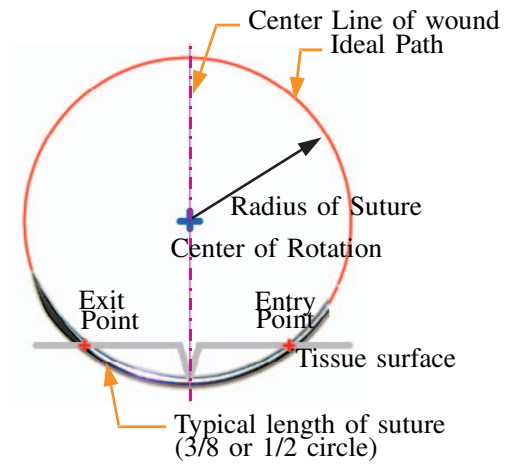

Fig. 10. Ideal path of needle tip for minimal tissue tear is a circle with the same radius as the suture needle and centered at needle center

and Cartesian velocities in (12) can be approximated by $\Delta \mathbf{x}_{g}^{w} / \Delta T=\mathbf{J}_{x s} \Delta \mathbf{s} / \Delta T$. We have our servo loop running at $1 \mathrm{KHz}$ and the optimized control algorithm loop running at $0.1 \mathrm{KHz}$. The JHU modular control library (CISST) - a $\mathrm{C}++$ library providing object oriented interface to real-time tasks and hardware, numerical methods and vector algebra, is used for programming the robot. In our setup, there are 86 constraints in optimization problem in (25b) and with are $2 \mathrm{GHz}$ Pentium IV, the average time to solve for each iteration is $1.6 \mathrm{~ms}$.

We performed two sets of experiments to validate our results. In the first set we used the readings of the motor encoders and the direct kinematics to determine the motion/position of the suture. In the second set of experiments we used the Optotrak to measure the pose of the end disk of the SLU and determine the motion of the suture. As mentioned in Section III-A, for our setup the suturing motion simplifies to a rotational motion about the center of end disk. Thus it suffices to consider the position for the center of end disk during the entire motion. A large error in the center would indicate the tip of the needle has strayed away from the desired path and is considered as undesirable motion. Figure 10 shows the ideal path of the needle tip that would result in minimal tissue tear.

\section{B. Experimental Results Based on Encoder Readings and Direct Kinematics}

We performed suturing motions using our system and recorded the encoder positions of the robot joints for approximately 180 seconds. We then used the forward kinematics of the hybrid system to determine the position of the center of suture. Fig. 11 shows the $\mathrm{X}, \mathrm{Y}$, and $\mathrm{Z}$ components of error, $\mathbf{p}_{g}^{w}-\mathbf{p}_{g}^{w}$ start as computed by the forward kinematic model in (10) using values provided by the motor encoders. This figure represents the motion of the suture center caused by the control algorithm and the robot controller while assuming an ideal SLU (i.e., no deflections and backlash). All the weights and control parameters for the redundancy resolution algorithm were the same as the ones used in our simulations. It is seen that the center of the suture is constrained within a sphere of radius $0.8 \mu \mathrm{m}$. The $\mathrm{X}, \mathrm{Y}$, and $\mathrm{Z}$ components of the error are 
very small and indicate that our high-level control algorithm generates a path that tracks the desired motions accurately.

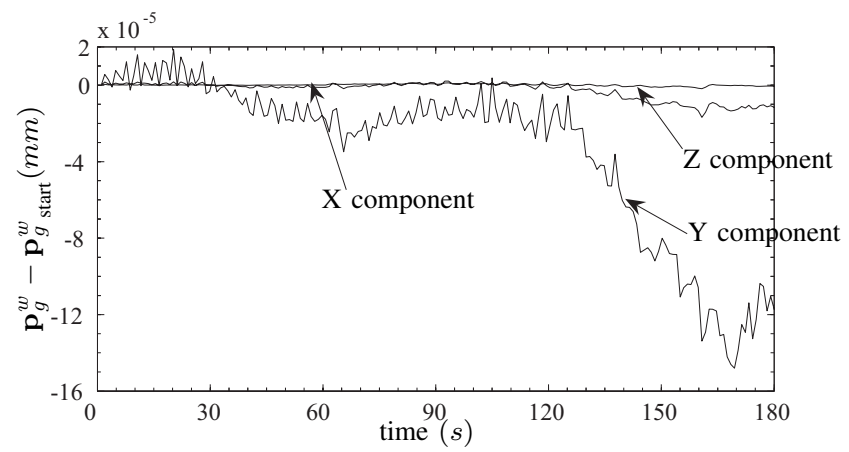

Fig. 11. $\mathrm{X}, \mathrm{Y}$, and $\mathrm{Z}$ components of $\mathbf{p}_{g}^{w}-\mathbf{p}_{g}^{w}$ start computed using motor encoder readings, where $\mathbf{p}_{g}^{w}$ is position of the gripper frame in world coordinates at any given instant, and $\mathbf{p}_{g}^{w}{ }_{\text {start }}$ is the position of the gripper frame at the beginning of suture biting motion.

\section{Experimental Validation Based on Direct Measurements of the Optotrak}

To further validate our results, we used an independent measurement to verify that the gripper frame was rotating about the axis $\hat{\mathbf{z}}_{\mathrm{g}}$ that passes through the center of the suture. We selected the Optotrak 3020 from Northern Digital Inc. (Waterloo, Canada) to provide optical measurement of the gripper frame.

The instrumentation consisted of two rigid bodies, one attached to the end of the disk of SLU, such that the reference frame of the rigid body coincided with the origin of the gripper frame. The other body was attached to the base of the robot to provide a reference for measurements. We attached six active markers to the end disk of the SLU and rotated the SLU about an arbitrary direction (Fig. 9(b)). We used least-squares fitting to determine the positions of these markers with respect to the gripper frame by fitting the $3 \mathrm{D}$ position readings of these markers to their respective circles of motion. Once these positions were determined we performed suturing motion and measured the 3D motion of the center of the suture. Data was collected for approximately 180 seconds with an average sampling rate of $5 \mathrm{~Hz}$.

Fig. 12 shows the $\mathrm{X}, \mathrm{Y}$, and $\mathrm{Z}$ components of $\mathbf{p}_{g}^{w}-\mathbf{p}_{g_{\text {start }}}^{w}$ as measured by Optotrak. The center of the suture is constrained in a sphere having a radius $1.84 \mathrm{~mm}$. As expected the errors measured by Optotrak are higher than measured by encoders alone, because of a number of factors that influence overall accuracy. The main cause of error seems to be the backlash in the large scale mock-up SLU. Also large scale mock-up model of the SLU is much more compliant than the $4 \mathrm{~mm}$ SLU because of the dimensions of its backbones compared to its overall length and diameter $(0.9 \mathrm{~mm}$ backbone diameter, $45 \mathrm{~mm}$ length, and $27.6 \mathrm{~mm}$ SLU diameter). The other cause of error is the inability to accurately determine the home configuration of the SLU in which all secondary backbones are equal in length to the central backbone. We plan to eliminate this source of error by providing absolute position measurements using potentiometers in the $4 \mathrm{~mm}$ SLU. Moreover, the accuracy of the Optotrak tracking system $(0.1 \mathrm{~mm}, 1 \mathrm{deg})$ also contributes to the overall error.

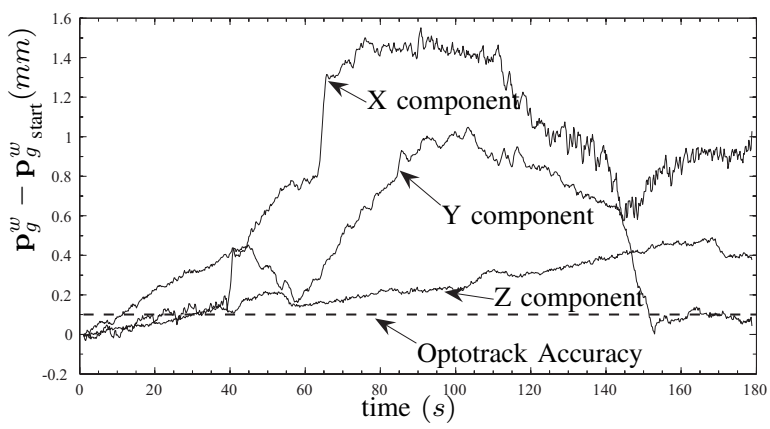

Fig. 12. $\mathrm{X}, \mathrm{Y}$, and $\mathrm{Z}$ components of $\mathbf{p}_{g}^{w}-\mathbf{p}_{g}^{w}$ start measured by Optotrak 3020 from Northern Digital Inc.(Accuracy $0.1 \mathrm{~mm}, 1 \mathrm{deg}$ ), where $\mathbf{p}_{q}^{w}$ is position of the gripper frame in world coordinates at any given instant, and $\mathbf{p}_{g}^{w}$ istart the position of the gripper frame at the beginning of suture biting motion.

\section{CONCLUSION}

This paper presented the kinematic modeling and high level control of a hybrid high DoF robots used for dexterous applications such as suturing in confined spaces. The high level control is based on a linearized multi-objective constrained optimization that is easily extendable to include additional constraints such as collision avoidance, anatomic-based constraints [22] and joint limits. The optimization problem is solved numerically at rates compatible with real-time control. The problem of suturing in confined spaces was formulated as a redundancy resolution and trajectory planning problem compatible with the optimization framework.

We have performed successful validation of our high level control using an experimental 8-DoF robot, composed from a 6 DoF LARS robot and a large-scale model of a Snake-Like Unit (SLU) used for distal dexterity. Simulations comparing suturing using the SLU verses a rigid tool to hold the suture were presented. These results show that the motions of the proximal joints are minimized if the distal dexterity is provided by the SLU. This is a crucial requirement for suturing in confined spaces, such as MIS of the throat. These simulations were validated by experiments that determined the efficacy of our high-level control and correctness of our kinematic modeling. These experiments were performed based on encoder readings and the forward kinematic model of the hybrid robot and compared to independent readings from an optical tracking device (Optotrak).

These results serve for validation of our high-level control and electronic hardware to be used in the final version of a tele-robotic system for MIS of the throat.

\section{ACKNOWLEDGEMENTS}

This work was partially funded by the National Science Foundation (NSF) under Engineering Research Center grant \#EEC9731478, NSF grant \#IIS9801684, and by the Johns 
Hopkins University internal funds. The authors also acknowledge the contribution of Peter Kazanzides in designing the low-level electronic hardware and software for this system.

\section{REFERENCES}

[1] Y. Wakahara, T. Tsuchihashi, and K. Asano, "A Computer Aided Manipulation System for a Multijoint Inspection Robot," in Proc. of the 32nd Conf. on Remote Syst. Tech., 1984, pp. 33-38.

[2] M. Takahashi, I. Hayashi, N. Iwatsuki, K. Suzumori, and N. Ohki, "The development of an in-pipe microrobot applying the motion of an earthworm," in 5th International Symposium on Micro Machine and Human Science, 1994, pp. 35-40.

[3] L. Phee, D. Accoto, A. Menciassi, C. Stefanini, M. C. Carrozza, and P. Dario, "Analysis and Development of Locomotion Devices for the Gastrointestinal Tract," IEEE Trans. Biomed. Eng., vol. 49, no. 6, pp. 613-616, 2002.

[4] K. Osuka and H. Kitajima, "Development of Mobile Inspection Robot for Rescue Activities: MIORA," in IEEE/RSJ Intl. Conf. on Intell. Robot. and Sys., 2003, pp. 3373-3377.

[5] A. Wolf, H. B. Brown, R. Casciola, A. Costa, M. Schwerin, E. Shamas, and H. Choset, "A Mobile Hyper Redundant Mechanism for Search and Rescue Tasks," in IEEE/RSJ Intl. Conf. on Intell. Robot. and Sys., 2003, pp. 2889-2895

[6] L. Ascari, C. Stefanini, A. Menciassi, S. Sahoo, P. Rabischong, and P. Dario, "A New Active Microendoscope for Exploring the Subarachnoid Space in the Spinal Cord," in Proc. IEEE Intl. Conf. Robot. and Automat., 2003, pp. 2657-2663.

[7] P. Breedveld and S. Hirose, "Development of the Endo-Periscope for improvement of depth perception in laparoscopic surgery," in 2001 ASME Design Engineering Technical Conferences Computers and Information in Engineering Conference, Pittsburgh, PA, USA, 2001.

[8] M. C. Montesi, B. Martini, A. Pellegrinetti, P. Dario, L. Lencioni, and A. Montano, "An SMA-base flexible active endoscope for minimal invasive surgery," Journal of Micromechanics and Microengineering, vol. 5, pp. 180-182, 1995.

[9] P. Dario, C. Paggetti, N. Troisfontaine, E. Papa, T. Ciucci, M. C. Carrozza, and M. Maracci, "A Miniature Steerable End-Effector for Application in an Integrated System for Computer-Assisted Arthroscopy," in Proc. IEEE Intl. Conf. Robot. and Automat., 1997, pp. 1573-1579.

[10] K. Ikuta, K. Yamamoto, and K. Sasaki, "Development of Remote Microsurgery Robot and New Surgical Procedure for Deep and Narrow Space," in Proc. IEEE Intl. Conf. Robot. and Automat., 2003, pp. 1103 1108.

[11] J. Piers, D. Reynaerts, H. V. Brussel, G. D. Gersem, and H. T. Tang, "Design of an Advanced Tool Guiding System for Robotic Surgery," in Proc. IEEE Intl. Conf. Robot. and Automat., 2003, pp. 2651-2656.

[12] N. Simaan, R. Taylor, and P. Flint, "A Dexterous System for Laryngeal Surgery," in Proc. IEEE Intl. Conf. Robot. and Automat., 2004, pp. 351357
[13] N. Simaan, R. Taylor, and P. Flint, "High Dexterity Snake-like Robotic Slaves for Minimally Invasive Telesurgery of the Upper Airway," in MICCAI, 2004, pp. 17-24.

[14] N. Simaan, "Snake-like Units Using Flexible Backbones and Actuation Redundancy for Enhanced Miniaturization," in Proc. IEEE Intl. Conf. Robot. and Automat., 2005, pp. 3023-3028.

[15] Y. Nakamura, H. Hanafusa, and T. Yoshikawa, "Task-Priority Based Redundancy Control of Robot Manipulator," Intl. J. Robot. Res., vol. 6 , no. 2, pp. 3-15, 1987.

[16] C. Klein and B. E. Blaho, "Dexterity Measures for the Design and Control of Kinematically Redundant Manipulators," Intl. J. Robot. Res., vol. 2, no. 6, pp. 72-83, 1987.

[17] J. M. Hollerbach and K. C. Suh, "Redundancy Resolution of Manipulators through Torque Optimization," IEEE Trans. Robot. Automat., vol. 3, no. 4, pp. 308-316, 1987.

[18] Y. Nakamura and H. Hanafusa, "Optimal Redundancy Control of Robot Manipulators," Intl. J. Robot. Res., vol. 6, no. 1, pp. 32-42, 1987.

[19] G. Chirikjain and J. Burdick, "The Kinematics of Hyper-Redundant Robot Locomotion,' IEEE Trans. Robot. Automat., vol. 6, no. 11, pp. 781-793, 1995.

[20] S. Sen, B. Dasgupta, and A. K. Mallik, "Variational approach for singularity-free-path planning of parallel manipulators," Mechanism and Machine Theory, vol. 38, pp. 1165-1183, 2003.

[21] J. Funda, R. Taylor, B. Eldridge, S. Gomory, and K. Gruben, "Constrained Cartesian Motion Control for Teleoperated Surgical Robots," IEEE Trans. Robot. Automat., vol. 12, no. 3, pp. 453-465, 1996.

[22] M. Li and R. Taylor, "Spatial Motion Constraints in Medical Robot Using Virtual Fixtures Generated by Anatomy," in Proc. IEEE Intl. Conf. Robot. and Automat., 2004, pp. 1270-1275.

[23] R. Taylor, J. Funda, B. Eldridge, S. Gomory, K. Gruben, D. LaRose, M. Talamini, L. Kavoussi, and J. Anderson, "A Telerobotic Assistant for Laparoscopic Surgery," IEEE Eng. Med. Biol. Mag., vol. 14, no. 3 pp. 279-288, 1995

[24] B. Eldridge, K. Gruben, D. LaRose, J. Funda, S. Gomory, J. Karidis, G. McVicker, R. Taylor, and J. Anderson, "A Remote Center of Motion Robotic Arm for Computer Assisted Surgery," Robotica, no. 14, pp. 103-109, 1996

[25] I. Gravagne and I. Walker, "Kinematic Transformations for RemotelyActuated Planar Continuum Robots," in Proc. IEEE Intl. Conf. Robot. and Automat., 2000, pp. 19-26

[26] C. L. Lawson and R. J. Hanson, Solving Least Squares Problems. Prentice-Hall, Inc., 1974.

[27] T. Yoshikawa, Foundations of Robotics. The MIT Press, 1990.

[28] A. Kapoor, N. Simaan, and P. Kazanzides, "A System for Speed and Torque Control of DC Motors with Application to Small Snake Robots," in Proc. IEEE Intl. Conf. Mechatron. and Robot., 2004 